\title{
A Case of Syringolymphoid Hyperplasia with Follicular Mucinosis
}

\author{
Emily L. Behrens ${ }^{a}$ Christine Jabcugad Jerad M. Gardner ${ }^{b, c}$ \\ Sareeta R. Parkere Douglas C. Parkere, ${ }^{\mathrm{f}}$ \\ ${ }^{a}$ College of Medicine, and Departments of ${ }^{b}$ Pathology and ${ }^{\mathrm{C}}$ Dermatology, University of \\ Arkansas for Medical Sciences, Little Rock, Ark., dDepartment of Pathology, Mayo Clinic, \\ Rochester, Minn., and Departments of ${ }^{\mathrm{e}}$ Dermatology and ${ }^{\mathrm{f}}$ Pathology, Emory University, \\ Atlanta, Ga., USA
}

\section{Key Words}

Syringolymphoid hyperplasia · Follicular mucinosis - Benign process · Lymphoproliferative disorder

\begin{abstract}
Syringolymphoid hyperplasia (SLH) is an extremely rare histopathological entity with fewer than 40 cases reported in the literature. SLH have been seen as both benign lesions and in association with T-cell lymphoproliferative lesions. A 20 -year-old male presented with a solitary, infiltrated plaque on the left cheek initially diagnosed as a sebaceous carcinoma at an external institution. A repeat biopsy demonstrated prominent follicular mucinosis (FM), squamous metaplasia of the eccrine coils, and a moderately dense perieccrine lymphocytic infiltrate mimicking eccrine carcinoma. The lesion was subsequently diagnosed as SLH with associated FM, an entity that has been previously reported in 12 cases, including this current case. This case highlights the characteristic features of a rare entity, emphasizes the potential for misdiagnosis of SLH, and adds to the current series of SLH described in the literature.
\end{abstract}

(C) 2016 The Author(s)

Published by S. Karger AG, Basel

\section{Introduction}

Syringolymphoid hyperplasia (SLH) is characterized by eccrine epithelial hyperplasia with squamous metaplasia and a dense lymphocytic infiltrate surrounding and infiltrating the ductal epithelium. First reported in 1969, SLH remains a very rare condition with less than 40 cases reported in the literature $[1,2]$. A subset of cases is associated with follicular mucinosis (FM); 12 cases of SLH with associated FM have been reported in the literature including this current case [1-7]. 
Fig. 1. Initial biopsy (made at an external institution). Multiple epithelial nests, some of which show ductal differentiation, are scattered through the dermis. There is a dense dermal lymphocytic infiltrate. Expansion of the overlying follicular infundibula is also seen. H\&E. $\times 20$.

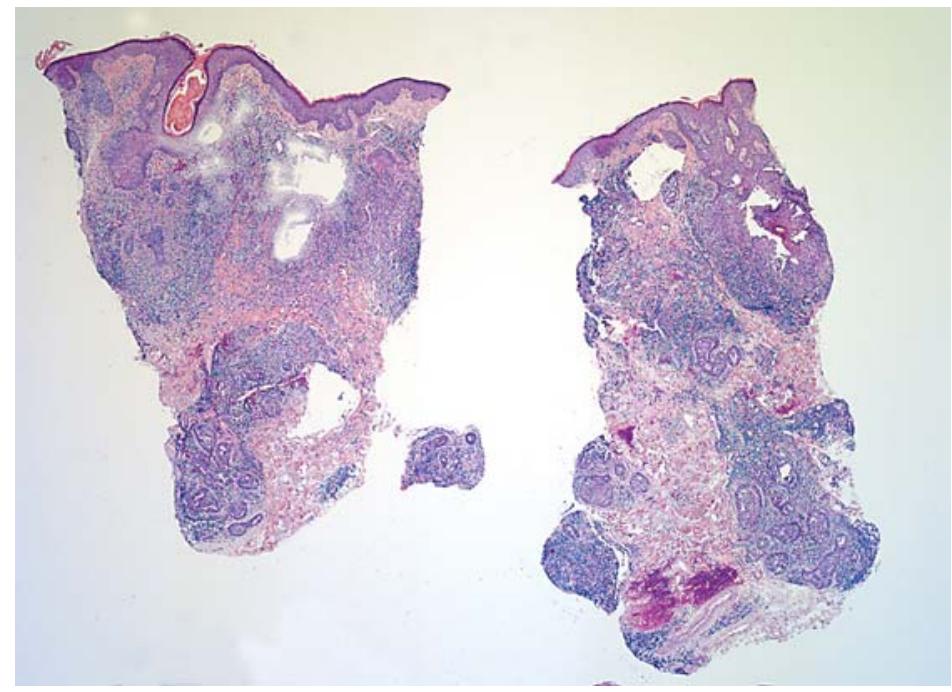

SLH occurs predominantly in males, and the vast majority of cases present with alopecia [8]. The spectrum of clinical features reported for SLH includes a solitary plaque, well-circumscribed patches with erythema or hypopigmentation, and associated alopecia and/or anhidrosis. The lesions may be localized or generalized [1, 2]. Follicular punctate erythema, if present, is thought to be a characteristic clinical finding that correlates with syringotropic lymphocytic infiltration [5]. Associated histopathological features include eccrine epithelial hyperplasia with squamous metaplasia, FM, and a perieccrine lymphocytic infiltrate which may exhibit pilotropism and/or epidermotropism [1]. T-cell clonality by T-cell receptor (TCR) gene rearrangement analysis has been reported in 23 cases [1]. Responses to treatments such as psoralen and ultraviolet A light, superficial localized radiotherapy, topical steroids, and systemic chemotherapy are variable and may be dependent upon the extent of involvement and underlying immunosuppressive states $[1,3,5]$.

\section{Case Report}

A 20-year-old male presented with a 4-year history of an asymptomatic $6 \times 4$-cm solitary, erythematous plaque with focal areas of pitting and prominent follicular orifices on his left infraocular cheek, extending to the nasal sidewall. The initial biopsy diagnosis from an external institution was sebaceous carcinoma. The patient was then referred to our institution for surgical management.

The initial skin biopsy performed at an external institution showed dermal nests of epithelial cells with moderate nuclear atypia displaying features suggestive of infiltrative growth with foci of ductal differentiation and an associated dense lymphocytic infiltrate (fig. 1, 2). The overlying follicular infundibula were also expanded and surrounded by a lymphocytic infiltrate. Submitted immunohistochemistry for cytokeratin 7 was diffusely positive in the dermal epithelial cells, and there was focal positivity for p63 (fig. 3). Although features of sebaceous carcinoma (diagnosis made at an external institution) were not identified, the prominent ductular structures in the dermis raised concern for an incompletely sampled eccrine carcinoma (fig. 2).

A repeat biopsy demonstrated prominent expansion of follicular infundibula with intrafollicular mucin, squamous metaplasia of the eccrine coils, and a moderate perieccrine 
Fig. 2. Initial biopsy (made at an external institution). Duct formation is clearly seen within the nests. The epithelial cells display moderate atypia. The dense lymphocytic infiltrate is composed of small lymphocytes, which are closely associated with, and focally infiltrate into, the epithelial nests. H\&E. $\times 100$.

Fig. 3. Initial biopsy (made at an external institution). Epithelial nests strongly express cytokeratin 7 by immunohistochemistry. $\times 40$.

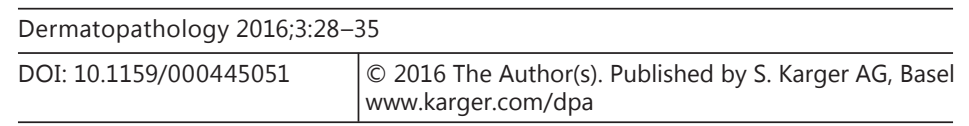

Behrens et al.: A Case of Syringolymphoid Hyperplasia with Follicular Mucinosis
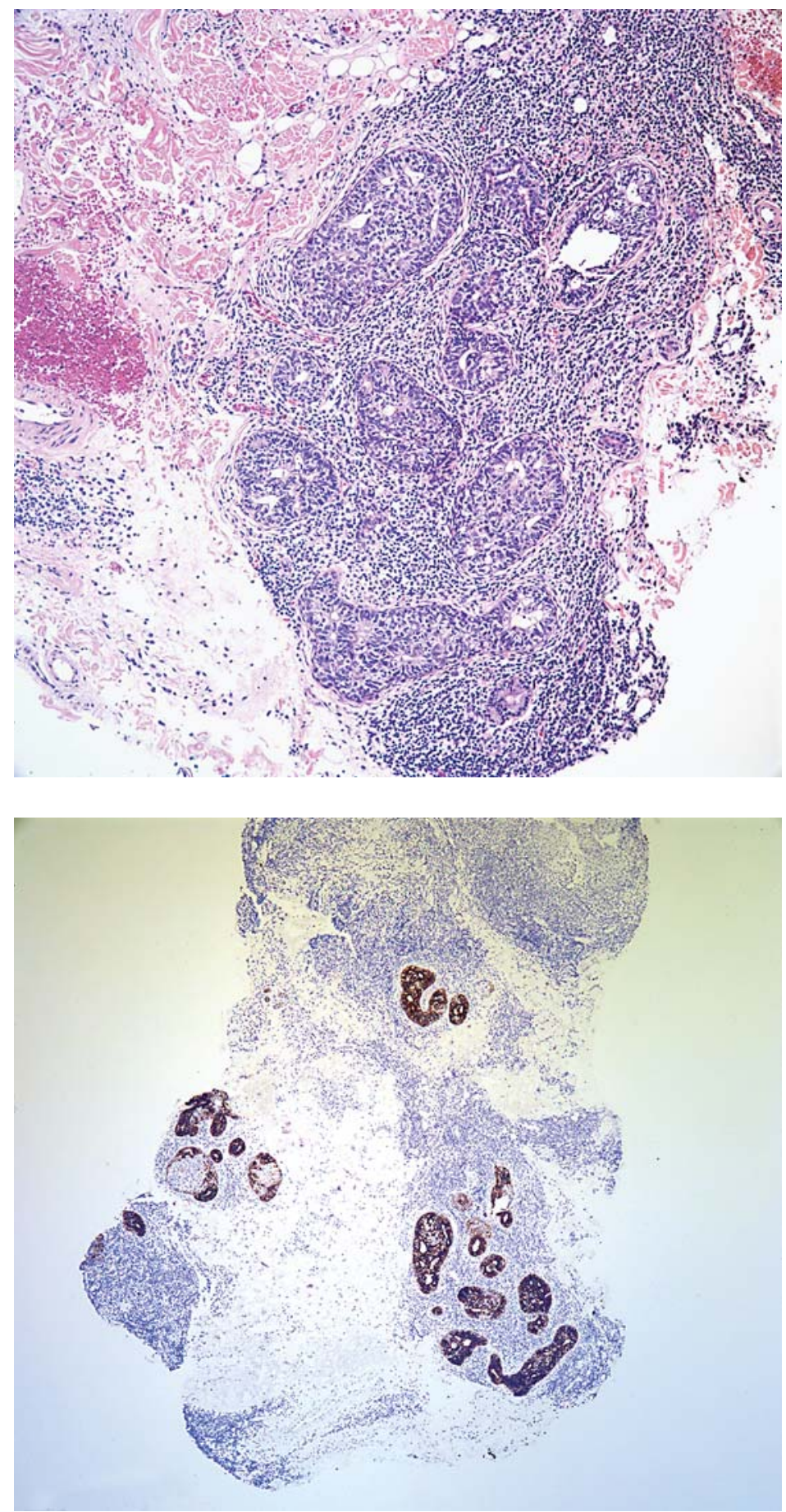

lymphocytic infiltrate (fig. 4-6). Lymphocyte atypia was not identified in either the initial or repeat biopsy. In view of the prominent FM and metaplastic eccrine changes, the lesion was diagnosed as SLH with associated FM. Immunohistochemistry showed that the majority of the dermal lymphocytic infiltrates consisted of CD3-positive T cells that showed a marked CD4 predominance (fig. 7, 8). TCR gene rearrangement studies were not performed.

Based on the clinical presentation, the young age of the patient, and the absence of lymphocyte atypia in the dermal infiltrate, the lesion was diagnosed as SLH with associated 
Fig. 4. Subsequent biopsy at our institution shows similar features. Clustered epithelial nests are present in the dermis, there is follicular expansion, and a dense lymphocytic infiltrate is present around the follicles and epithelial nests. H\&E. $\times 20$.

Fig. 5. Subsequent biopsy. Closer inspection reveals the epithelial nests to actually be proliferative eccrine glands with marked syringometaplasia. The altered eccrine coils are surrounded and focally infiltrated by small lymphocytes. H\&E. $\times 100$.

\begin{tabular}{l|l}
\hline Dermatopathology 2016;3:28-35 \\
\hline DOI: 10.1159/000445051 & $\begin{array}{l}\text { @ 2016 The Author(s). Published by S. Karger AG, Basel } \\
\text { www.karger.com/dpa }\end{array}$ \\
\hline
\end{tabular}

Behrens et al.: A Case of Syringolymphoid Hyperplasia with Follicular Mucinosis
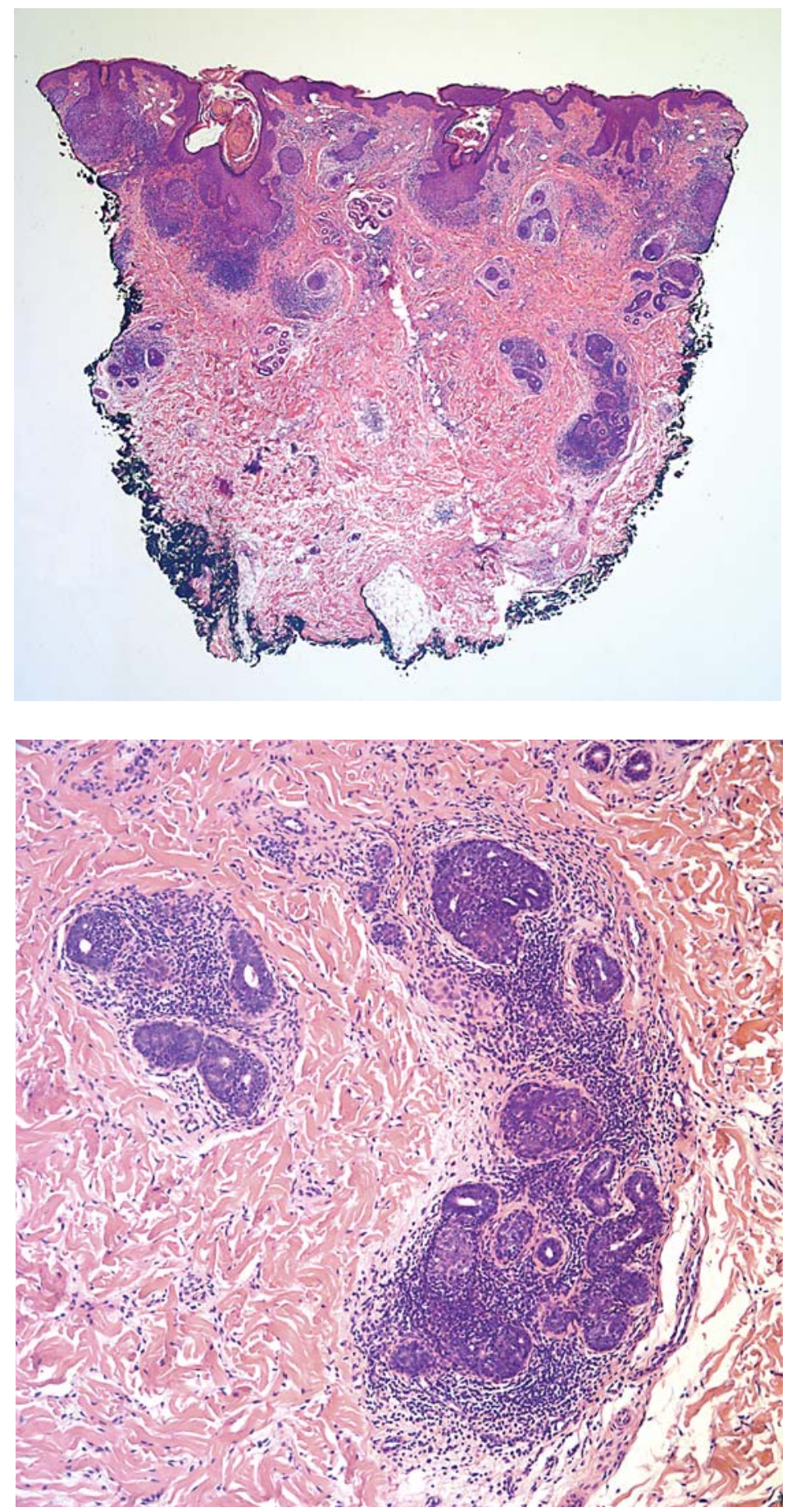

FM. Therapy with hydroxychloroquine $400 \mathrm{mg}$ daily for 4 months was initiated. However, no clinical improvement was observed, and he developed similar additional lesions on the right intraocular cheek, right anterior thigh, and left lower back. He was subsequently lost to follow-up. Although the clinical and histopathological features at presentation were suggestive of a primary, benign variant of SLH with associated FM, long-term clinical follow-up is essential to exclude adnexotropic mycosis fungoides. 
Fig. 6. Subsequent biopsy. A second punch biopsy performed at the same time showed FM characterized by follicular expansion and distention by mucin between individual follicular epithelial cells. H\&E. $\times 100$.

Fig. 7. Subsequent biopsy. The periadnexal lymphocytes are almost exclusively small $\mathrm{T}$ cells as highlighted by this CD3 immunohistochemical stain $(\times 40)$. No obvious epidermotropism is identified in the overlying epidermis.

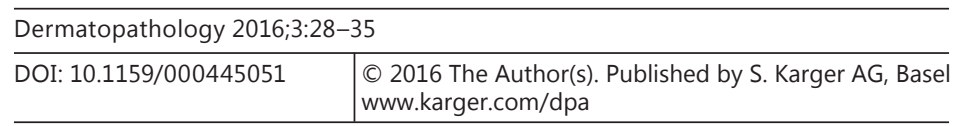

Behrens et al.: A Case of Syringolymphoid Hyperplasia with Follicular Mucinosis
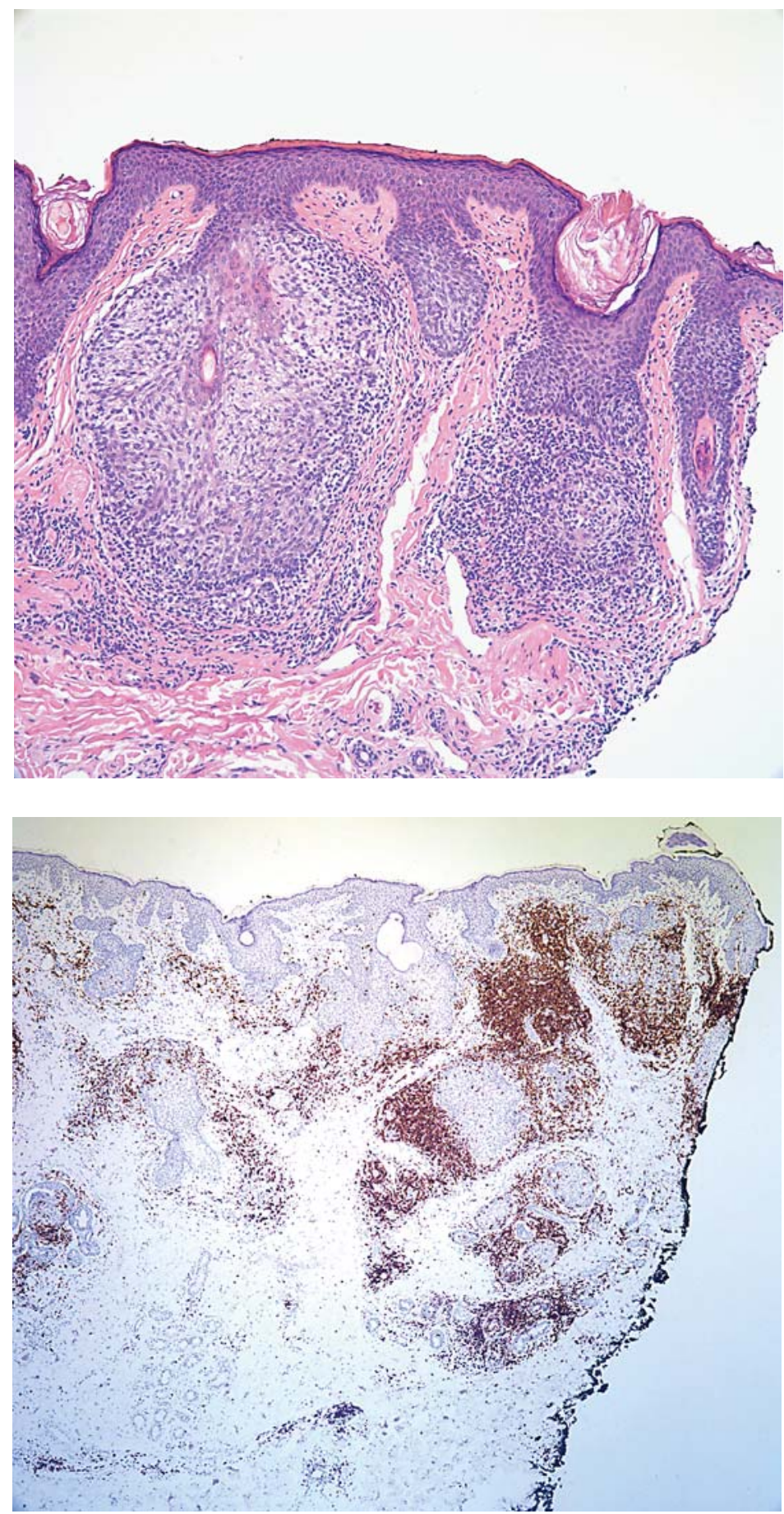

\section{Discussion}

Most patients with SLH have been previously misdiagnosed clinically or histopathologically. Eccrine syringometaplasia (eccrine squamous syringometaplasia) is an epithelial reaction pattern characterized by proliferation of the eccrine ductal epithelium, typically displaying squamous or, less commonly, mucinous features [9]. While eccrine syringometaplasia is not uncommon, an associated dense lymphocytic infiltrate is rare. Eccrine syringo- 
Fig. 8. Subsequent biopsy. The T cells are closely associated with the metaplastic eccrine coils, and focal infiltration of the ductal epithelium is also seen. CD3 immunostain. $\times 100$.

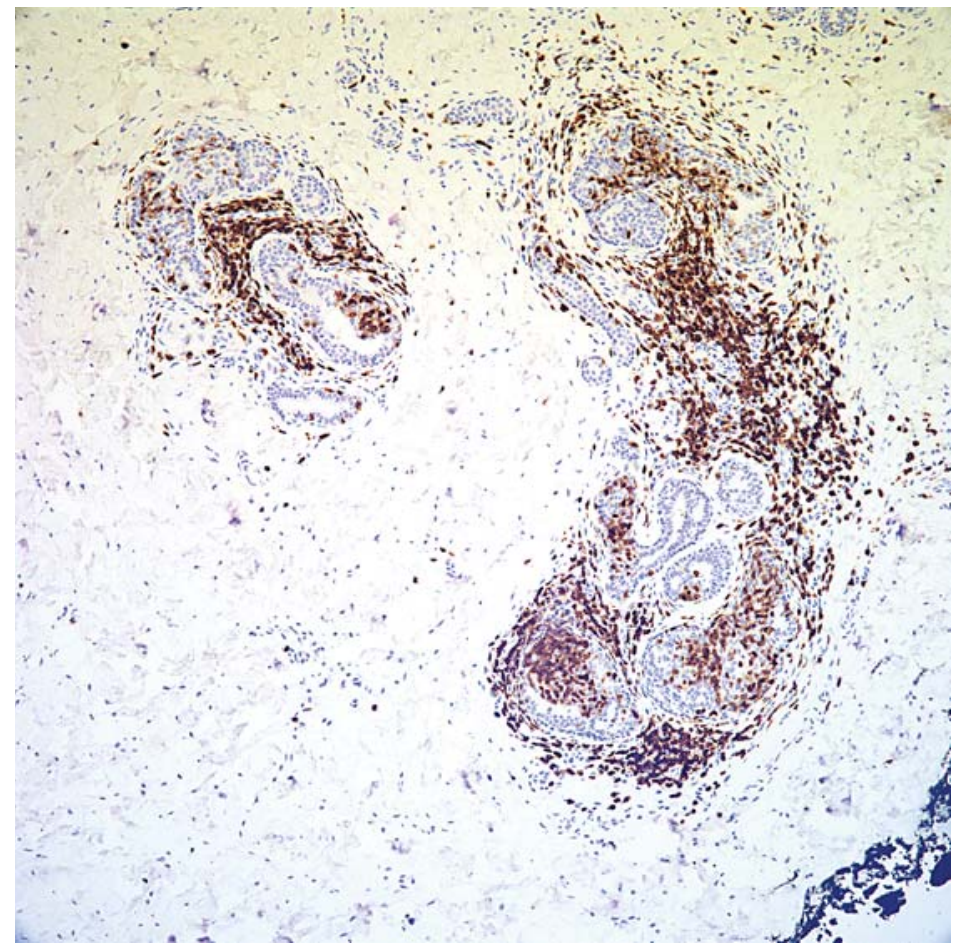

metaplasia is a potential diagnostic pitfall that may be misdiagnosed as a sweat gland carcinoma or squamous cell carcinoma depending on the extent of the squamous metaplasia present. Correlation with the clinical history, recognition of the preserved lobular pattern of eccrine glands, and the absence of overlying epidermal atypia are critical in distinguishing eccrine syringometaplasia from invasive carcinoma [9]. In 2001, Haller et al. [3] described the first female patient affected with a localized variant of SLH. In 2003, Hobbs et al. [7] described 2 cases of SLH, 1 with FM as seen in our case, and the other shown to be positive for TCR gamma chain gene rearrangement. In 2011, Vijayashree et al. [5] reported the youngest case of SLH to date, a 12-year-old boy from India who was initially misdiagnosed and treated for Hansen's disease.

Thein et al. [1] concluded that TCR gene analysis of lesional skin and peripheral blood may be a prognostic indicator of disease progression based on their 8 cases of SLH with alopecia in which TCR gene analysis of skin and/or blood was performed [1,5]. Hobbs et al. [7] suggested that $\mathrm{T}$-cell clones in peripheral blood were mirrored by progressive skin lesions. Langerak et al. [10] suggested that clonality assessment is warranted for samples that remain clinically suspect and/or histomorphologically unclear. While a polyclonal pattern detected in Ig/TCR multiplex PCR assays is useful in confirming most reactive lesions, clinical followup with new tissue sampling should be considered to reach a final diagnosis in cases with suspicious Ig/TCR clonality results [10]. In retrospect, T-cell clonality studies in our case may have provided additional diagnostic support for benign lesions to rule out mycosis fungoides. Fortunately, most of the reported cases of SLH seem to have an indolent and nonprogressive course $[1,7]$.

The etiology of SLH remains controversial, and several concepts have been postulated. Burg and Schmöckel [6], Tannous et al. [4], Thein et al. [1], and Pileri et al. [2] speculated that SLH represents a syringotropic variant of cutaneous T-cell lymphoma. However, Haller et al. [3] hypothesized that SLH is a syringotropic variant of FM and should be viewed as a facul- 
tative precursor lesion of mycosis fungoides. Huang et al. [8] suggested a possible autoimmune pathogenesis for the T-cell infiltrate in their patient, a 28-year-old woman who presented with acquired generalized anhidrosis and a history of subclinical Sjögren's syndrome. Although the specific cellular mechanisms to induce eccrine syringometaplasia have not been identified, it has numerous etiologic associations. Inflammation is the most common association, but it has also been linked to herpes virus infections and several chemotherapeutic drugs, most recently BRAF inhibitors vemurafenib and dabrafenib [9, 11-15]. Based on 7 patients with a mean follow-up of 10 years, Brown et al. [16] postulated that primary FM is a clonal disorder with limited or benign cutaneous manifestations.

Psoralen and ultraviolet A light, localized radiotherapy, and/or topical steroids are initial therapeutic considerations. When evidence of systemic involvement is present, systemic chemotherapy may be required [1]. The female patient in the study of Haller et al. [3] was refractory to UV treatment, systemic prednisone, cyclophosphamide, and various antibiotics [3]. Schneider et al. [17] reported hydroxychloroquine as an effective treatment in their 6 cases of idiopathic FM. They observed complete remission without development of lymphoma in 3-23 years of follow-up. Therapy with hydroxychloroquine was initiated in our patient without clinical response.

To our knowledge, this case of SLH with associated FM is only the twelfth reported case of this rare entity. SLH have been seen as both benign lesions and in association with T-cell lymphoproliferative lesions [1-9]. Long-term clinical and histopathological follow-up may be necessary to more accurately classify this rare lesion and assess its prognostic features.

\section{Statement of Ethics}

The manuscript was prepared in compliance with all ethical and confidentiality guidelines and principles.

\section{Disclosure Statement}

The authors have no conflicts of interests to declare.

\section{References}

1 Thein M, Ravat F, Orchard G, Calonje E, Russell-Jones R: Syringotropic cutaneous T-cell lymphoma: an immunophenotypic and genotypic study of five cases. Br J Dermatol 2004;151:216-226.

2 Pileri A, Facchetti F, Rütten A, Zumiani G, Boi JS, Fink-Puches R, Cerroni L: Syringotropic mycosis fungoides: a rare variant of the disease with peculiar clinicopathologic features. Am J Surg Pathol 2011;35:100-109.

3 Haller A, Elzubi E, Petzelbauer P: Localized syringolymphoid hyperplasia with alopecia and anhidrosis. J Am Acad Dermatol 2001;45:127-130.

4 Tannous Z, Baldassano MF, Li WV, Kvedar J, Duncan LM: Syringolymphoid hyperplasia and follicular mucinosis in a patient with cutaneous T-cell lymphoma. J Am Acad Dermatol 1999;41:303-308.

5 Vijayashree R, Kumar A, Nott A, Rao R: Syringolymphoid hyperplasia with alopecia and anhidrosis in a 12-year-old boy: a case report from rural south India. Int J Dermatol 2011;50:1552-1554.

6 Burg G, Schmöckel C: Syringolymphoid hyperplasia with alopecia - a syringotropic cutaneous T-cell lymphoma? Dermatology 1992;184:306-307.

7 Hobbs JL, Chaffins ML, Douglass MC: Syringolymphoid hyperplasia with alopecia: two case reports and review of the literature. J Am Acad Dermatol 2003;49:1177-1180.

8 Huang C, Kuo T, Chan H: Acquired generalized hypohidrosis/anhidrosis with subclinical Sjögren's syndrome: report of a case with diffuse syringolymphoid hyperplasia and lymphocytic sialadenitis. J Am Acad Dermatol $1996 ; 35: 350-352$. 
9 Serrano T, Saez A, Moreno A: Eccrine squamous syringometaplasia. A prospective clinicopathologic study. J Cutan Pathol 1993;20:61-65.

10 Langerak AW, Groenen PJ, Brüggemann M, et al: EuroClonality/BIOMED-2 guidelines for interpretation and reporting of Ig/TCR clonality testing in suspected lymphoproliferations. Leukemia 2012;26:2159-2171.

11 Chetty R, Bramdev A, Govender D: Cytomegalovirus-induced squamous syringometaplasia. Am J Dermatopathol 1999;21:487-490.

12 Hurt MA, Halvorson RD, Petr, FC, et al: Eccrine squamous syringometaplasia. A cutaneous sweat gland reaction pattern in the histologic spectrum of chemotherapy-associated eccrine hidradenitis and neutrophilic eccrine hidradenitis. Arch Dermatol 1990;126:73-77.

13 Chemotherapy-related bilateral dermatitis associated with eccrine squamous syringometaplasia. Reappraisal of epidermiological, clinical and pathological features. J Am Acad Dermatol 2011;64:1092-1103.

14 Lescoat A, Droitcourt C, Stock N, et al: Vemurafenib-induced eccrine squamous syringometaplasia. Dermatology 2013;226:362-364.

15 Liuti F, Martin PA, Montenegro Damaso T, et al: Eccrine squamous syringometaplasia associated with dabrafenib therapy. J Am Acad Dermatol 2013;69:273-274.

16 Brown HA, Gibson LE, Pujo RM, Lust JA, Pittelkow MR: Primary follicular mucinosis: long-term follow up of patient younger than 40 years with and without clonal T-cell receptor gene rearrangement. J Am Acad Dermatol 2002; 47:856-862.

17 Schneider SW, Metze D, Bonsmann G: Treatment of so-called idiopathic follicular mucinosis with hydroxychloroquine. Br J Dermatol 2010;163:420-423. 